\title{
The cost implications of surveillance of ICU infections
}

\section{Farhad Kapadia}

\section{Introduction}

Epidemiological data is necessary in the evaluation and management of ICU associated infections. Our institute periodically collects systematic data regarding a specific site of potential infection to evaluate the incidence of these problems. The aim of the current analysis is to evaluate the cost implications in obtaining the incidence, microbiology and outcome of ICU associated infections.

In the last 2 years, at various intervals we have prospectively collected data regarding central venous catheter, arterial catheter, urinary catheter, ventilator associated infections and bacteremia in the ICU. We report the incidence of these infections, and the cost needed to evaluate this incidence.

\section{Methods and Materials}

For vascular catheters, all those inserted in the study month (March 2001 for CVCs and April 2001 for arterial catheters) were included. Only those catheters suspected of being clinically infected were studied. The catheter tip was cultured by Maki's method and a simultaneous blood culture was collected.

For urinary catheters, paired urine samples were collected for routine and microbiological analysis at the time of catheter insertion and removal in April and May 2002. Pyuria was diagnosed if a spun down urine sample had $>5 \mathrm{WBC} / \mathrm{HPF}$. Bacteuria was diagnosed if the urine sample had $>100,000 \mathrm{CFU} / \mathrm{ml}$. Clinical urinary tract infection was diagnosed if, along with pyuria and bactiuria, the patient had fever and leucocytosis which had no other obvious cause.

\section{From:}

Hinduja National Hospital \& Medical Research Center. Mumbai

Correspondence:

Consultant Intensivist, Hinduja Hospital \& Research center, Mumbai, India. E-mail: fkapadia@vsnl.com
For ventilator associated pneumonia, NIH criteria were used for diagnosis. The study was done for a period of 6 months from June to November 2002. Only patients ventilated for more than 48 hours were included.

For bacteremia, all blood cultures sent for an 8 months period (March to Oct 2002) were analyzed.

\section{Results}

Central Venous Catheters: A total of 122 catheters were inserted in March 2001 of which 24 were clinically considered at risk of infection. Of the 24 catheters studied 0 were colonized, 5 were infected and none had an associated bacteremia. One had a dual bacterial infection. The duration of use of these five catheters were $8,8,9$, 16 and 29 days respectively. 3 were inserted in the ICU, 1 in the OT and 1 on the general floors. 1 of the patients had the same organism growing at a remote site suggesting an endogenous seeding of the catheter. The organisms were, Pseudomonas aerogenosa (3 isolates), Klebsiela pneumonea (2 isolates) and Enterobacter agglomerans ( 1 isolate). The Cost Implications were as follows. Each catheter culture cost Rs.190/- and each blood culture cost Rs. 470/- during the study period. The actual cost was Rs. 15,840/-, which was borne by patient. If a complete surveillance had been done the additional cost would have been Rs. $64,680 /-$, borne by the hospital, giving a total project cost Rs. $80,520 /$ - for 1 month data.

Arterial Catheters: A total of 102 catheters were inserted in April 2001 of which 18 were clinically considered at risk of infection. Of the 18 catheters studied 2 were colonized, 3 were infected and 2 had an associated bacteremia. The duration of use of these five catheters were $10,10,14,16$ and 25 days respectively. 4 of these 5 catheters were femoral while one was radial. 3 were inserted in the ICU and 2 in the OT. 3 of the patients had the same organism growing at a remote site 
suggesting an endogenous seeding of the catheter. The organisms were, Pseudomonas aerogenosa (2 isolates), Klebsiela pneumonea (2 isolates) and Enterobacter agglomerans ( 1 isolate). The Cost Implications were as follows. The actual cost was Rs. 11,880/- which was borne by patient. If a complete surveillance had been done the additional cost would have been Rs. 55,440/-, borne by the hospital, giving a total project cost Rs. $67,320 /-$ for 1 month data.

Urinary catheters: Paired urine samples were collected for routine and microbiological analysis at the time of catheter insertion and removal in April and May 2002 from 100 consecutive patients. At the time of catheter insertion 26 patients had pyuria, and none had bacteuria or clinical urinary tract infection. At the time of catheter removal 29 patients had pyuria. 6 of these had significant bacituria of whom 2 had fever and leucocytes, which could have represented a clinical urinary tract infection. The organisms were, E Coli (2 isolates), Klebsiela pneumonea (2 isolates) and Enterobacter agglomerans (2 isolate). The Cost Implications were as follows. The cost of the Urine CS + RM samples was Rs. $98,000 /-$ and the cost of salary of research fellow was Rs. 8,000/-. The whole expense of Rs. $1,07,000 /$ - for two months data was borne by the research society of the hospital.

Ventilator associated Pneumonia: Data was collected from patients ventilated for more than 48 hours between June and November 2002. 92 patients were ventilated for a total of 1409 days. There were 33 episodes of VAP in 26 patients. The organisms were as follows: Pseudomonas aerogenosa $=10$, E Coli=8, Klebs Pneumonia $=7$, Candida albicans $=4$, Klebs oxytoca $=2$, Acenetobacter $=2$, Enterobacter $=2$, Klebs oxanae $=1$, Enterococci $=1$, Staph aureus $=1$, Candida species $=1$ and aspergillous $=1$. In the patients who developed VAP $m$ the mortality was $16 / 26$, while in those without VAP it was $36 / 66$. The difference was not statistically significant $(P=0.54)$. The cost of a tracheal culture was Rs. 290/-, a blood count Rs. 280/- and a chest $X$ ray was Rs. 260/- The actual cost of the diagnosis of 33 episodes of VAP was Rs. 27,720/-. This cost was epidemiological data was borne by the patient. The projected cost for collecting data every 3 ventilated days per patients would be Rs. 5,84,735/-.
Bacteremia: An analysis of all positive cultures was done for a period of 8 months between March $1^{\text {st }}$ and Oct $31^{\text {st }} 2002$. A total of 1485 patients were admitted to ICU in this period and 402 blood cultures were sent on clinical grounds in this period. There were 107 episodes of bacteremia in this period in 88 patients. The organisms were as follows: Klebs Pneumonie=30, E Coli=13, Enterobacter species=11, Pseudo aerogenosa=10, Candida species $=8$, Staph aureus=7, Enterococci=6, Staph albus $=6$, A $\ldots=4$, Klebs species $=3$, A baumani=3, Strep species $=3$, Candida albicans=2, C. .diversus.. $=2$, Pseudo Cepacea $=1$, Pseudo species $=1$, Klebs Oxytocia $=1$ and $M$ morgagni=1. The mortality in this group was $45 / 88$ compared to $96 / 1418$ in those patients admitted in the same period in whom there was no bacteremia. The difference in mortality was highly significant $(P=0.00001)$. The cost of the blood culture was Rs. 470/- the cost of clinical surveillance of bacteremia in this 8 months period was Rs.1,88,940/-. The cost for this epidemiological data was borne by the patient.

The projected cost for a 12 month surveillance of vascular catheter infections, urinary catheter infections, VAPs and bacteremia would be Rs. 38,68,960/- (CVC= $9,66,240+A L=8,07,840$, Urinary cath $=6,42,000 /$, $+\mathrm{VAP}=11,69,470,+$ Bacteremia $=2,83,410$ )

\section{Discussion}

Collection and interpretation of data regarding ICU infection faces problems. The diagnosis of the infection is dependent of definitions, which in turn are dependent on both clinical and microbiological criteria. For a true incidence, all patients of study period should be included in denominator, not just those with clinical suspicion of infection. This exercise needs a great degree of manpower and is costly. Data from a limited period may not be representative, necessitating data over substantial periods of time.

Given these limitations, we noted incidence of these infections to similar to those quoted in the standard international literature. The organisms were predominantly gram negative bacteria at all sites studied. Bacteremia was associated with a higher mortality.

The actual expenses in our data collection exercise were significant at Rs. 3,51,388/- for relative limited 
study periods. The annual projected cost of an adequate surveillance was Rs. 38,68,960/-. This expense may not be warranted in a developing country. Ambiguity in interpreting the results of this data further raises doubt as to the value of this exercise. Due to these factors, widespread use of ICU infections surveillance may not be appropriate in developing countries. 